\title{
MusicCohort: Cross-sectional feasibility study of an assessment protocol for student musicians
}

\section{Julius Bruder \\ University of Alberta \\ Nikolaus Ballenberger \\ Hochschule Osnabruck \\ Bethany Villas \\ University of Alberta \\ Charlotte Haugan \\ University of Alberta \\ Kimiko McKenzie \\ University of Alberta \\ Zalak Patel \\ University of Alberta \\ Amynah Mevawala \\ University of Alberta}

\section{Christoff Zalpour}

Hochschule Osnabruck

Christine Guptill ( $\sim$ guptill@ualberta.ca )

University of Alberta https://orcid.org/0000-0002-5080-2672

\section{Research}

Keywords: Musicians, music students, assessment protocol, validation, mental health, physical health

Posted Date: May 22nd, 2020

DOI: https://doi.org/10.21203/rs.3.rs-29065/v1

License: (c) (i) This work is licensed under a Creative Commons Attribution 4.0 International License.

Read Full License 


\section{Abstract}

This study examined the feasibility of employing an assessment battery developed in Germany, investigating mental and physical health of university music students, in Canada. Using a cross sectional design, first-semester music and control students were recruited at two university campuses. Eligibility criteria were: 1) first-semester, full-time undergraduate music major (case) or in another university program (control), 2) over the age of 16 . Exclusion criteria were: 1) diagnosis of neurological, orthopaedic or psychological condition, 2) diagnosis of infection or systemic disease, 3) regular consumption of medication for pain or mental health diagnosis, 4) varsity athlete, 5) for controls only, enrollment in music courses where a grade is assigned for music performance (e.g. studio lessons). Both groups completed questionnaires and physical testing, including range of motion, core strength and pressure pain threshold. Data for 19 music and 50 control students were analysed. Musician participants demonstrated tendencies towards poorer mental and physical health. This German protocol is feasible in a Canadian university setting. Canadian music students demonstrate similar mental and physical health outcomes to those in the literature and in the parent study. The results of this feasibility study should be confirmed in a larger study.

\section{Background And Objectives}

Music has been part of human life and culture for all of recorded history (1). Most of us connect music particularly listening - with joy and awe for the musician's creativity, perfection and accuracy when they perform incredibly difficult pieces. The long and challenging path that musicians must take to get to the top tends to be overlooked.

In the research literature, Playing Related Musculoskeletal Diseases (PRMD) are primarily examined in professional musicians, where prevalence rates typically range between $70-80 \%$ (9-11). Studies focusing on music students' playing-related health also suggest elevated stress and anxiety level in children and tertiary students $(12,13)$. Furthermore, repetitive strain injuries in children and tertiary students have been found to be as high as $90 \%$ (12-14). In comparison, $66 \%$ of Canadian youth aged 12-19 injure themselves while engaging in sports (15). In this journal, Stanhope et al. (2019) suggested that more consistency is needed in research of musician's PRMD, including the development of more valid tools specific to the task (16).

Given these high prevalence rates, it is important to determine risk factors connected to the development of PRMD, and to develop consistent and reliable assessment methods. To this end, the University of Applied Sciences Osnabrück has launched a longitudinal study, monitoring the health of music students throughout their entire degree. The research team developed a detailed assessment protocol to monitor the student's health over this period (16). 
The goal of the present study was to replicate the assessment protocol developed in the German longitudinal study and test its feasibility in a Canadian music program. In particular, we aimed to ensure that processes and management were reproducible and that all necessary resources were available (17). Feasibility in the Canadian educational system would allow for transcultural comparison of health in music students due to standardized procedures. A secondary goal was to examine the prevalence of health conditions in Canadian music students at the beginning of their university studies as compared to non-music students.

\section{Subjects And Methods}

\section{Study design}

For this feasibility study, a cross-sectional design was selected. Participants were first-semester undergraduate students at the University of Alberta, Edmonton, Canada. They were tested once without follow-up. Inclusion criteria were: 1) enrolled in first-semester as a full-time undergraduate student majoring in music (case) or in any other faculty at the University of Alberta (control), 2) voluntary participation, 3) over the age of 16 . Exclusion criteria were: 1) diagnosis of neurological, orthopaedic or psychological condition, 2) diagnosis of infections or systemic disease, 3) regular consumption of pain medication or medication for mental health diagnosis, 4) varsity athlete, 5) for controls only, enrollment in selected courses (e.g. studio lessons) from the music department, where a grade is assigned for playing an instrument.

Recruitment and testing took place from 2016 until 2018, each semester between September and November for 2016-18 fall cohorts (3), and between March and May for the 2018 spring cohort (1). Seventy-nine students contacted the researchers to indicate interest in participation. Data from ten participants were excluded for the following reasons: a non-music student taking music courses; a varsity athlete; participants with mental health diagnoses; participants choosing to withdraw without stating reasons; and participants lost to follow-up. Data from a total of 69 students were included in the analysis, of which 19 were music students and 50 were non-music/control students. No sample size calculation was conducted for this feasibility study. Ethics approval for this study was obtained from the Research Ethics Boards at the Osnabrück University of Applied Sciences and the University of Alberta.

This feasibility study used the following procedure: students were recruited through posters displayed across campus, on social media (Twitter and Facebook), through a campus-wide email distribution system, and via flyers distributed in-person in a first-year music class. Potential participants were 
informed that they would receive a $\$ 5$ coffee gift card for participation, plus one additional gift card for referring additional eligible participants.

Initial eligibility of participants was confirmed via email. Eligible participants were then invited to be tested at the investigator's lab on campus. On testing day, the procedure, as well as inclusion and exclusion criteria, were explained to the participant. Eligible participants were then asked to provide written informed consent. Testing started with participants completing a baseline questionnaire asking general demographic information and history of pain and musculoskeletal complaints. This was followed by additional questionnaires assessing self-reported mental and physical well-being. The second part of the protocol included physical testing of range of motion (ROM), mechanosensitivity, core strength, and general mobility. At the end of the assessment, participants were briefed about their performance. Overall, testing took approximately 70 minutes. All assessments were performed by an investigator who was either a trained physiotherapist, occupational therapist or an occupational therapy student. Every investigator received a minimum of 2 hours of instruction/training for the assessments.

This pilot study adhered as closely as possible to the study protocol used by Ballenberger et al. to allow for comparability and to test feasibility of the protocol in a different geographical and cultural setting (16). Furthermore, through continuous communication with the authors of the parent study in Germany, refinements to the original protocol were reflected in our study protocol at the earliest convenience. This paper describes the final protocol used. Changes are discussed in the discussion.

\section{Assessment of physical and mental health}

The original participant questionnaire was translated from German into English by a native German speaker who is fluent in both languages. This study aimed to compare music and non-music students. In the initial cohort, music students answered a playing-related pain questionnaire. Neither music nor control students, however, were asked to answer any questions about general musculoskeletal pain prevalence. For better comparison, 20 questions about general pain were added before recruitment of the third cohort. Those questions were based on the general pain questions used in the German parent study.

\section{Health-related Quality of life}

To screen health-related quality of life, we used the RAND 12 questionnaire, which is free and does not require a license. This makes the test more user-friendly for replication of the study and future implementation in clinical settings. Furthermore, a custom scoring algorithm that is more appropriate for 
chronic conditions was employed, which does not rely on the basic assumption that physical and mental health component scores are not related (18). Test-retest reliability is reported to be excellent (ICC=0.78) and adequate $(I C C=0.60)$ for physical and mental health composites, respectively (19).

Initially, the parent study in Germany used the Stress-Coping-Inventory (SCI) (16). An optional, more versatile, mental health screening questionnaire was found in the 21-item Depression Anxiety Stress Scales (DASS-21), which - unlike the SCI - has also been translated into multiple languages. The DASS21 screens and distinguishes between the three mental health dimensions of depression, anxiety and stress (20). It demonstrates good internal consistency of 0.82-0.97, for both clinical and non-clinical populations $(21,22)$.

\section{Core endurance}

Core strength was assessed using four tests: a full plank, lateral planks on the right and left sides, and the Biering-Sørensen test for extensor endurance (23). Both the Biering-Sørensen test and plank tests have excellent inter-rater reliability $(I C C=0.8-0.99$ and $I C C=0.95$, respectively) $(23,24)$. Investigators demonstrated the correct positioning and allowed participants to try. Participants were then asked to hold each position until fatigued or needing to stop for any other reason. Other reasons participants in this study gave for stopping included pain, discomfort or vigorous shaking. A 30 second break between planks was given to eliminate confounding fatigue. Any position change was verbally corrected once; any further correction led to termination of the test.

\section{General mobility.}

To assess general mobility/ hypermobility, the Beighton score and the Sitting-Rising Test (SRT) were utilized. The Beighton score assigns a score of 0 to 9 reflecting general mobility (25). Participants performed four movements bilaterally, which were assessed by the investigator. The SRT assesses general mobility out of 10-points. Participants were asked to sit cross-legged on the floor, and rise with minimal support $(26,27)$. For any additional support needed, points were deducted from a 10-point scale. Both tests have good intra- and inter-rater reliability $(26 ; 28)$.

Kenny Music Performance Anxiety Inventory.

Music Performance Anxiety was evaluated using the Kenny Music Performance Anxiety Inventory (KMPAl-R). The inventory was first developed in 2004 and is the only validated tool that not only measures cognitive, behavioral, and physiological aspects of performance anxiety, but also "underlying 
psychological vulnerability" (29). The latest, revised version consists of 40 items, subdivided into eight domains. A participant can score a maximum of 240 points, where a higher score indicates more severe MPA symptoms. Questions address playing-related anxiety and the influence of different external sources. It has shown excellent internal consistency (Cronbach's Alpha $=0.94)(30)$. The questionnaire is musician-specific, and was therefore only answered by music students.

\section{Mechanosensitivity.}

To test mechanical pressure pain threshold, the same nine bilateral testing points as the original protocol by Ballenberger et al. were assessed (16). For the first cohort, a mechanical algometer (Pain Diagnostics \& Thermography, Italy) was used, which was then exchanged for a digital model (FDIX Wagner Instruments, Greenwich, CT, USA). Each point was tested three times, with a recovery period of 5 seconds in between.

Playing-related Musculoskeletal Disorders and pain/complaints interfering with daily living

Musicians answered the Musculoskeletal Pain Intensity and Interference Questionnaire for Musicians (MPIIQM). The tool, containing 13 questions, evaluates musicians' current playing-related health concerns (31). It generates two sub scores for pain interference (0-40) and pain intensity (0-50) (32). There are currently no cut-off scores established for this tool. It has adequate internal consistency and test-retest reliability (31).

\section{Statistical Analysis}

IBM SPSS, version 25, and Microsoft Excel 2013 were used to analyse collected data. Alpha was set at $p<0.05$. Most characteristics were not normally distributed, so non-parametric tests were chosen, using Mann-Whitney $\mathrm{U}$ test and the Chi-square-test for continuous and categorical variables, respectively. A preliminary analysis showed that musician and control groups were comparable in terms of both age and gender, and therefore, data were not adjusted for these variables. Effect size $r$ was calculated by dividing the z-value by square root of $\mathrm{N}(33)$.

\section{Results}

\section{General demographics}

Between October 2016 and November 2018, 19 music and 50 control students were tested and included in data analysis. Gender distribution of participants was similar in both 
groups with $68.4 \%$ female among musician participants, and $62 \%$ female in the control group.

Differences in the baseline characteristics between musicians and controls were statistically significant for both nutrition $(p=0.022)$ and physical activity $(p=0.001)$. Musicians' self-reported scores were lower for both of these health behaviours than controls. Participants were grouped into those who reported physical activity (PA) at/above the suggested 90 minutes per week (34), and those who reported lower PA. Analysis showed that musicians were significantly less likely to engage in PA $\left(\chi^{2}=6.25, p=0.016\right.$, OR 4.20). For the remaining characteristics, both groups were comparable (see table 1).

The average course load in hours per week (excluding labs) for musicians and controls was $18.87 \pm 3.3$ and $18.638 \pm 5.20$, respectively. In addition, musicians spent an average of 24.67 hours playing either their primary or secondary instrument.

\section{Table 1. General demographics*}




\begin{tabular}{|c|c|c|c|c|}
\hline & Total & Musicians & Control & Statistics \\
\hline Total & \begin{tabular}{|l|}
69 \\
\end{tabular} & 19 & 50 & \\
\hline Female gender & $44(63.8 \%)$ & $13(68.4 \%)$ & $31(62 \%)$ & $\begin{array}{l}\mathrm{p}=0.781 \\
\mathrm{X}^{2}=0.246\end{array}$ \\
\hline Age, yrs & $18.00(2)$ & $18.00(2.00)$ & $18.00(0)$ & $\begin{array}{l}\mathrm{p}=0.480 \\
\mathrm{r}=-0.085 \\
\mathrm{Z}=-0.707\end{array}$ \\
\hline Height, cm & $164.5(0.12)$ & $164.5(12.00)$ & $166.5(13.00)$ & $\begin{array}{l}\mathrm{p}=0.961 \\
\mathrm{r}=-0.049 \\
\mathrm{Z}=-0.049\end{array}$ \\
\hline Body weight, kg & $59.5(10.50)$ & $59.5(10.5)$ & $61.00(13.75)$ & $\begin{array}{l}\mathrm{p}=0.829 \\
\mathrm{r}=-0.216 \\
\mathrm{Z}=-0.216\end{array}$ \\
\hline$\overline{\mathrm{BMI}}$ & $21.77(5.30)$ & $21.77(3.06)$ & $21.70(2.85)$ & $\begin{array}{l}\mathrm{p}=0.565, \\
\mathrm{r}=-0.070 \\
\mathrm{Z}=-0.578\end{array}$ \\
\hline Sleep duration, $\mathrm{hr}$ & $7.00(0.63)$ & $7.00(0.63)$ & $7.00(1.44)$ & $\begin{array}{l}\mathrm{p}=0.309 \\
\mathrm{r}=-0.124 \\
\mathrm{Z}=-1.028\end{array}$ \\
\hline Course hours/ wk & $17.75(5.80)$ & $17.75(5.80)$ & $18.00(4.00)$ & $\begin{array}{l}\mathrm{p}=0.941 \\
\mathrm{r}=-0.010 \\
\mathrm{Z}=-0.080\end{array}$ \\
\hline Physical activity & $1.25(2.80)$ & $1.25(2.8)$ & $4.00(4.00)$ & $\begin{array}{l}\mathrm{p}=0.001 * \\
\mathrm{r}=-0.391 \\
\mathrm{Z}=-3.249\end{array}$ \\
\hline Alcohol glasses/wk & $0.25(1.30)$ & $0.25(1.3)$ & $0.00(0.90)$ & $\begin{array}{l}\mathrm{p}=0.321 \\
\mathrm{r}=-0.123 \\
\mathrm{Z}=-1.002\end{array}$ \\
\hline Nutrition & $6.00(1.30)$ & $6.00(1.30)$ & $7.00(2.00)$ & $\begin{array}{l}\mathrm{p}=0.022^{*} \\
\mathrm{r}=-0.274 \\
\mathrm{Z}=-2.281\end{array}$ \\
\hline $\begin{array}{l}\text { Instrument Playing hours } \\
\text { (musicians only) }\end{array}$ & NA & 21.75 (18.38) & $\mathrm{N} / \mathrm{A}$ & $\mathrm{N} / \mathrm{A}$ \\
\hline
\end{tabular}


*Values represent as median and Interquartile Range or frequency and percentage for each respective group, test statistics for Man-Whitney-U-Test, $\alpha=0.005$, Bold * show $\alpha<0.005, r=$ effect size

\section{Core strength}

Musicians demonstrated lower core strength, with all but the full plank test times being significantly different between groups. It is noteworthy that the control group also performed on average below the norm in all four endurance tests (35).

\section{Table 2. Core endurance}

\begin{tabular}{|c|c|c|c|c|}
\hline Test & Total* & Musician* & Control* & Statistics \\
\hline Right side plank & $49.00(45.00)$ & $30.00(37.00)$ & $56.50(38.64)$ & $\begin{array}{l}\mathrm{p}=0.008^{*} \\
\mathrm{r}=-0.315 \\
\mathrm{Z}=-2.614\end{array}$ \\
\hline Left side plank & $49.00(40.50)$ & $29.00(35.00)$ & $53.50(34.25)$ & $\begin{array}{l}\mathrm{p}=0.054 \\
\mathrm{r}=-0.240 \\
\mathrm{Z}=-1.995\end{array}$ \\
\hline Full plank & $69.00(60.00)$ & $52.00(67.00)$ & $74.00(55.75)$ & $\begin{array}{l}\mathrm{p}=0.046^{*} \\
\mathrm{r}=-0.232 \\
\mathrm{Z}=-1.928\end{array}$ \\
\hline Biering-Sorensen-test & $111.00(69.00)$ & $80.00(69.00)$ & $123.00(49.50)$ & $\begin{array}{l}\mathrm{p}=0.009 * \\
\mathrm{r}=-0.313 \\
\mathrm{Z}=-2.599\end{array}$ \\
\hline
\end{tabular}

*Values represent median and Interquartile Range, statistics for Mann-Whitney-U-test, $\alpha=0.005, \quad$ Bold * shows $\alpha<0.005 r=$ effect size

Mechanosensitivity 
Mechanosensitivity testing showed that musicians had lower mean pain thresholds for every testing point. A Mann-Whitney $U$ test revealed statistically significant difference in mean mechanosensitivity (of all measuring points) for musicians and controls, respectively $(p=0.029)$. Gender-specific differences were present, with females having consistently lower mean ranks, although these were not statistically significant. However, there was no clear pattern as to which testing points were significantly different.

Table 3. Mechanosensitivity, measured by mechanical pressure pain threshold 


\begin{tabular}{|c|c|c|c|c|}
\hline Test point & All $(\mathrm{N}=69) *$ & Musician $(\mathrm{N}=19)^{*}$ & Control $(\mathrm{N}=50) *$ & Statistics \\
\hline R Trapezius & $45.30(26.69)$ & $41.84(29.00)$ & $47.30(28.67)$ & $\begin{array}{l}\mathrm{p}=0.200, \\
\mathrm{r}=-0.155, \\
\mathrm{Z}=-1.290\end{array}$ \\
\hline L Trapezius & $42.17(33.40)$ & $37.10(17.49)$ & $46.48(35.41)$ & $\begin{array}{l}\mathrm{p}=0.050^{*}, \\
\mathrm{r}=-0.236, \\
\mathrm{Z}=-1.961\end{array}$ \\
\hline R Supraspinatus & $47.33(34.47)$ & $40.54(22.17)$ & $49.39(40.83)$ & $\begin{array}{l}\mathrm{p}=0.227, \\
\mathrm{r}=-0.147, \\
\mathrm{Z}=-1.216\end{array}$ \\
\hline L Supraspinatus & $44.70(28.89)$ & $35.00(26.21)$ & $51.09(32.41)$ & $\begin{array}{l}\mathrm{p}=0.008^{*}, \\
\mathrm{r}=-0.315, \\
\mathrm{Z}=-2.619\end{array}$ \\
\hline R Wrist Extensor & $39.40(17.88)$ & $30.40(15.53)$ & $40.59(17.14)$ & $\begin{array}{l}\mathrm{p}=0.023^{*}, \\
\mathrm{r}=-0.272, \\
\mathrm{Z}=-2.257\end{array}$ \\
\hline L Wrist Extensor & $31.20(19.36)$ & $29.00(11.20)$ & $35.50(22.01)$ & $\begin{array}{l}\mathrm{p}=0.184, \\
\mathrm{r}=-0.161, \\
\mathrm{Z}=-1.337\end{array}$ \\
\hline R Wrist Flexor & $51.50(28.86)$ & $41.68(27.40$ & $56.75(26.51)$ & $\begin{array}{l}\mathrm{p}=0.021 * \\
\mathrm{r}=-2.297, \\
\mathrm{Z}=-0.277\end{array}$ \\
\hline L Wrist Flexor & $40.83(23.08)$ & $33.70(19.94)$ & $42.77(22.06)$ & $\begin{array}{l}\mathrm{p}=0.030^{*}, \\
\mathrm{r}=-0.260, \\
\mathrm{Z}=-2.163\end{array}$ \\
\hline R Temporalis & $23.17(12.92)$ & $18.96(9.02)$ & $25.05(12.02)$ & $\begin{array}{l}\mathrm{p}=0.009 * \\
\mathrm{r}=-0.310, \\
\mathrm{Z}=-2.573\end{array}$ \\
\hline L Temporalis & $21.00(10.00)$ & $19.29(7.00)$ & $21.58(11.00)$ & $\begin{array}{l}\mathrm{p}=0.169, \\
\mathrm{r}=-0.167, \\
\mathrm{Z}=-1.384\end{array}$ \\
\hline Mean algometer & $37.43(15.28)$ & $30.53(11.20)$ & $38.76(16.29)$ & $\mathrm{p}=0.029 *$ \\
\hline
\end{tabular}




\begin{tabular}{|l|l|l|l|l|}
$\mathrm{r}=-0.265$ \\
$\mathrm{Z}=-2.190$ \\
\hline
\end{tabular}

*Values represent median and Interquartile Range in Newton, statistics for Mann-Whitney-U-test, $\alpha=0.005$, Bold * shows $\alpha<0.005, r=$ effect size

\section{Range of Motion}

Range of motion (ROM) was tested with the same strategy as implemented by Ballenberger et al. (16). There were no significant differences between musicians and controls on any of the ROM movements tested. Furthermore, both cohorts' ROM did not differ significantly from the norms.

\section{Table 4. Range of motion}

\begin{tabular}{|c|c|c|c|c|}
\hline Movement & All (N=69)* & Musician $(\mathrm{N}=19) *$ & Control $(\mathrm{N}=50) *$ & Statistics \\
\hline Mean CROM & $59.17(10.08)$ & $59.83(11.00)$ & $58.97(10.33)$ & $\begin{array}{l}\mathrm{p}=0.648, \\
\mathrm{r}=-0.077, \\
\mathrm{Z}=-0.643\end{array}$ \\
\hline Mean ROM & $74.44(8.25)$ & $74.31(7.31)$ & $74.69(8.55)$ & $\begin{array}{l}\mathrm{p}=0.486, \\
\mathrm{r}=-0.085, \\
\mathrm{Z}=-0.705\end{array}$ \\
\hline
\end{tabular}

*Values represent median and Interquartile Range of range of motion in degrees, test statistics for MannWhitney-U-test, Bold * shows $\alpha=0.005, r=$ effect size

\section{General mobility}

Hand span was not statistically different between musicians and controls and was similar to that of the general public for both samples. Although differences were not significant, 
musicians did have larger hand span for all four measurements (digit one-to-five and twoto-five on both hands). Both the Beighton score and Sitting-Rising Test did not show any significant differences between musicians and controls.

\section{Self-Reported Mental Health}

Musicians had poorer self-reported mental health scores in all three domains on the DASS21. Only on the stress domain, however, was this difference statistically significant $(p=0.043)$. Comparing female and male students, the scores for female students were lower; however, this difference was not statistically significant.

\section{Figure titles:}

Figure 1: Frequency (\%) of symptomatic participants by DASS21 domain.

For the Kenny Music Performance Anxiety Inventory (KMPAI-R), over half of the tested musicians (63.6\%) scored above the suggested cut-off of 84 (30), indicating more severe symptoms of Music Performance Anxiety, with a mean score of $94.27( \pm 56.64)$.

\section{Self-reported Quality of Life}

When compared to normative data for the SF-36 for Canadians of a similar age (36), the largest differences were observed in the following domains: Bodily Pain, Vitality, Role emotional, Mental Health and Mental Composite Score (MCS). However, these differences were not statistically comparable, since they employ two different questionnaires. When compared Differences between music students and controls were statistically significant for 
Bodily Pain $(\mathrm{p}=0.004)$ and approached statistical significance for General Health $(p=0.053)$. Mental Composite Scores were below the norm for both cohorts.

Table 5. RAND12 results for total sample and by cohort 


\begin{tabular}{|c|c|c|c|c|}
\hline RAND12 & Overall $(\mathrm{N}=69)$ & Musicians ( $\mathrm{N}=19)$ & Non-Musicians $(\mathrm{N}=50)$ & Statistics \\
\hline Physical functioning & $55.95(8.50)$ & $55.95(8.50)$ & $55.95(8.50)$ & $\begin{array}{l}p=0.560, \\
r=-0.560, \\
Z=-0.575\end{array}$ \\
\hline Role physical & $57.17(9.51)$ & $52.42(9.51)$ & $57.17(5.94)$ & $\begin{array}{l}\mathrm{p}=0.190, \\
\mathrm{r}=-0.159, \\
\mathrm{Z}=-0.190\end{array}$ \\
\hline Bodily pain & $57.27(9.41)$ & $47.86(9.41)$ & $57.27(0.00)$ & $\begin{array}{l}\mathrm{p}=0.004 * \\
\mathrm{r}=-0.348 \\
\mathrm{Z}=-2.887\end{array}$ \\
\hline General health & $55.49(10.38)$ & $45.11(10.38)$ & $55.49(10.38)$ & $\begin{array}{l}\mathrm{p}=0.053, \\
\mathrm{r}=-0.231, \\
\mathrm{Z}=-1.918\end{array}$ \\
\hline Vitality (energy) & $44.91(10.38)$ & $44.91(20.77)$ & $44.91(10.38)$ & $\begin{array}{l}p=0.170, \\
r=-0.167, \\
Z=-1.391\end{array}$ \\
\hline Social functioning & $56.17(10.12)$ & $56.17(10.12)$ & $56.17(10.12)$ & $\begin{array}{l}\mathrm{p}=0.459, \\
\mathrm{r}=-0.092, \\
\mathrm{Z}=-0.770\end{array}$ \\
\hline Role emotional & $44.98(17.34)$ & $39.20(17.34)$ & $44.98(13.00)$ & $\begin{array}{l}\mathrm{p}=0.175, \\
\mathrm{r}=-0.164, \\
\mathrm{Z}=-1.365\end{array}$ \\
\hline Mental health composite score & $48.99(10.76)$ & $44.68(14.67)$ & $54.74(5.97)$ & $\begin{array}{l}p=0.169, \\
r=-0.171, \\
Z=-1.424\end{array}$ \\
\hline Physical health composite score & $53.90(6.29)$ & $51.60(5.91)$ & $49.50(10.59)$ & $\begin{array}{l}\mathrm{p}=0.055 \\
\mathrm{r}=-0.231 \\
\mathrm{Z}=-1.921\end{array}$ \\
\hline
\end{tabular}

Values present median and Interquartile Range for RAND12 scores, test statistics for Mann-Whitney-U-test, $\alpha=0.005$. Bold * shows $\alpha<0.005$ 


\section{$\underline{\text { Pain/complaints }}$}

The point prevalence (7-day) of Playing-Related Musculoskeletal Disorder (PRMD) in this sample was $31.6 \%$, whereas lifetime prevalence was $68.4 \%$. Life-time-prevalence rate of $68.4 \%$ was also consistent with findings in the literature (9-14). The most prevalent playingrelated pain location, as recorded by the MPIIQM, was the right forearm $(\mathrm{N}=4)$. Right wrist, shoulder-neck region, and left and right hand $(\mathrm{N}=3$, respectively) were the next most frequently reported locations. The most common pain locations were similar to those reported in the literature (6-14). Participants reported a mean playing-related pain intensity of 4.06 on a Likert scale of 0 to 10 as measured by the MPIIQM. Pain interference with participants' ability to play at the level to which [they] were accustomed was reported as a mean of 8.53 on a scale of 0 to 10 .

\section{Feasibility}

The trial of the German assessment protocol in a Canadian educational setting was considered feasible. All tools were either available in English or were unvalidated demographic collection forms that were translated by the first author, who is fluent in both languages. Furthermore, all clinical tests were reproducible using the same procedures. This study highlighted recruitment as the main critical area in our setting for a successful and relevant reproduction. Despite differences in the university settings, we found that the protocol was feasible in both settings.

\section{Discussion}

Our results show that a protocol used for a longitudinal study in Germany/Europe is feasible to be reproduced in a Canadian university setting. In comparison to the German parent study, these cohorts presented with similar health profiles, although no in-depth comparative analysis has yet been conducted (37). Additionally, the data presents a clear trend that first year music students in a Canadian university have poorer mental and physical health than other, non-music first year students. 
Music students and control students reported very similar numbers of course hours per week. Music students, however, spent an average of 24.76 hours playing their instrument outside of classes. We speculate that this might be a contributing factor as to why music students spend significantly less time on physical activity. Long hours spent on school work and practicing, significantly less physical activity, significantly worse self-reported nutrition, as well as tendencies toward more alcohol consumption and less hours of sleep might all be contributing factors to worse physical and mental health in music students.

The point prevalence (7 day) of PRMD in this study, $31.6 \%$, was comparable to that reported in the literature (14). This is lower than in a comparative study by Berque and colleagues using this tool (38). Since their study examined PRMD in professional musicians, this difference may reflect variation between post-secondary student musicians and professionals.

It is striking that music students had a lower pressure pain threshold on all 18 testing points, as well as a higher mean mechanosensitivity $(p=0.029)$, while simultaneously reporting higher levels of stress and anxiety symptoms. A link between stress and pain threshold has previously been suggested, which may explain these results (39). Furthermore, testing points with statistically significant differences were located on the forearm, left supraspinatus and left trapezius. Those muscle groups are often recruited in instrumental performance $(40,41)$. The wrist extensor muscle wad was the most commonly reported location for PRMD, likely reflecting its action in the performance requirements of several instruments (41, 42).

With respect to performance anxiety, there are currently no validated cut-offs developed for the KMPAl-R. Kenny, however, suggests a preliminary cut-off of 84 points (35). The mean score of students in this sample was well above this score. However, the large variability in the KMPAI-R scores of participants in our study implies the need for a larger sample.

Self-reported mental and physical health, as captured by the RAND 12, were not statistically different between groups, except for the Bodily Pain domain. By contrast, compared to the Canadian age-related norm, the total sample population performed worse in all domains except Bodily Pain. Considered as a whole, the participants in this study performed above the norm in the domain Role Physical; however, musicians scored below the norm, while control students scored above the norm. These differences were not significant; however, the difference of 5.77 points between the MCS scores of musicians and controls was higher than the minimal clinically important difference in two patient populations $(43,44)$.Higher 
levels of stress and mechanosensitivity are also consistent with higher levels of Bodily Pain on the RAND 12.

The literature suggests that Physical Activity (PA) has a beneficial effect on pain threshold $(45,46)$. This is congruent with our sample, where musicians did significantly less PA and reported lower pain threshold. This finding must also be viewed with caution because the RAND 12 is a self-report measure. Kreutz et al. have demonstrated that music students' self-report of their health is more optimistic than objective findings (47). Clinicians must therefore be cautious when assessing musicians' health through self-report.

The research literature suggests gender difference in several of the measured outcomes. A preliminary analysis of gender differences showed a trend for females to present with poorer physical and mental health outcomes. These results were, however, not statistically significant and need to be studied further $(48,49)$.

In summary, a preliminary comparison of the Canadian data to the original longitudinal study in Germany showed similarities in student's health profiles, however, a larger sample and more research are needed (37).

\section{Study Limitations}

A limitation of this study is that the procedure had to be adapted throughout data collection. For example, it was discovered that in the parent study, all participants were being asked about general pain ratings. This was added later in our study, resulting in missing data for this parameter from earlier participants. Furthermore, unlike in the German study, the age cut off was adapted to 16 years of age. This age was chosen to capture situations where younger students were attending university (typically in Canada, they would be 18 or older), and were mature enough to provide informed consent.

After the first cohort, the investigators realized that a revised version of the Kenny Music Performance Inventory was being used in the German parent study. The protocol was then adapted to include the revised version. Hence, the eight students in the first cohorts completed an earlier version (KMPAI), for which no cut-off-scores are available (50). These participants' data were therefore excluded from data analysis concerning performance anxiety. 
Another limitation is the relatively small number of participants, which reduces statistical power. Recruiting music students proved to be more challenging than we had anticipated. There may be a number of explanations for this difficulty, including the length of time required for testing (70 minutes) and the fact that our lab was located a 15 minute walk away from the Department of Music. Participants in Germany were expected to take part in testing annually. Since health promotion and injury prevention are relatively new concepts in post-secondary music education in Canada, program culture and research ethics requirements meant we were reliant on the goodwill of students and a $\$ 5$ gift card incentive to encourage participation. A cultural change towards health promotion in schools of music in North America has been recommended by educators, researchers and clinicians (51), and this change is supported in Canada through curriculum guidelines that recommend health education (52).

This study is a helpful step in understanding differences between music student's and other student's health habits, and in understanding how Canadian music students compare to music students around the world. It also helps to highlight the difficulties in recruiting music students for research studies. Anecdotally, music students often tell clinicians and researchers that they are very busy, and our findings related to class and practice time seem to support this.

Thus far, our study has not included a longitudinal component, but this could be an important addition in future studies. Since the demands of different instruments require specific postures and may cause instrument-specific strain on the body, future studies may also examine instrument-specific risk factors. The results encourage further study in the area, but larger samples are needed for more meaningful conclusions.

In conclusion, our study has demonstrated that the protocol developed by our German colleagues is feasible in a Canadian university population. We have also demonstrated similar trends in Canadian postsecondary students as compared to German students. Future studies with larger sample sizes can provide further insight into the risk factors affecting post-secondary music students, providing a basis for prevention and intervention.

\section{References}

1. Wold MA. An Outline History of Western Music. 8th ed. Dubuque,USA: WCB Brown \& Benchmark; 1994. 
2. Rickert DL, Halaki M, Ginin KA, Barrett MS, Ackermann BJ. The use of fine-wire EMG to investigate shoulder muscle recruitment patterns during cello bowing: The results of a pilot study. Journal of Electromyography and Kinesiology. 2013; doi:10.1016/j.jelekin.2013.07.013.

3. Statistics Canada. The Cumulative Earnings of Postsecondary Graduates over 20 years: Results by Field of Study 2015. https://www150.statcan.gc.ca/n1/pub/11-626-x/11-626-x2014040-eng.htm. Accessed 29 Jun 2019.

4. A Statistical Profile of Artists in Canada in 2016 (With summary information about cultural workers). 2016. https://hillstrategies.com/resource/statistical-profile-of-artists-in-canada-in-2016/. Accessed 26 Jun 2019.

5. Statistics Canada. Economic Importance of Culture and Sport in Canada 2015. http://www.statcan.gc.ca/pub/13-604-m/2014075/culture-4-eng.htm. Accessed 26 Jun2019.

6. Chesky K, Devroop K. The effect of college music instruction, gender, and musician type on income from performing music. Med Prob Perf Art. 2003;18:72-77.

7. Brandfonbrener AG. Musculoskeletal problems of instrumental musicians. Hand Clinics. 2003; doi:1016/S0749-0712(02)00100-2.

8. Parry W. Introduction: The musician's hand. Hand Clinics. 2003; doi:0.1016/S0749-0712(03)00007-6.

9. Kok LM, Huisstede BMA, Voorn VMA, Schoones JW, Nelissen RGHH The occurrence of musculoskeletal complaints among professional musicians: A systematic review. Int Arch Occup Environ Healt. 2016; doi:10.1007/s00420-015-1090-6.

10. Fishbein M, Middlestadt S, Ottati V, Straus S, Ellis A. Medical problems among ICSOM musicians: Overview of a national survey. Med Probl Perform Art. 1988;3:1-8.

11. Ackermann B, Driscoll TR, Kenny DT. Musculoskeletal pain and injury in professional orchestral musicians in Australia. Med Probl Perform Art 2012; 27(4):181-187.

12. Boucher $H$, Ryan CA (2011) Performance stress and the very young musician. Journal of Research in Music Education. 2011; doi:1177/0022429410386965.

13. Spahn C, Richter B, Zschocke I. Health attitudes, preventive behaviour and playing-related health problems among music students. Med Prob Perform Art. 2002;17:22-28.

14. Ranelli SM. Prevalence of playing-related musculoskeletal symptoms and disorders in children learning instrumental music. Med Prob Perform Art. 2008;23:178-185.

15. Statistics Canada. Activity When Injured, By Age Group, Household, Population Aged 12 or Older, Canada, 2009 to 2010. 2015. http://www.statcan.gc.ca/pub/82-624-x/2011001/article/desc/1150602-desc-eng.htm. Accessed 26 Jun2019.

16. Ballenberger N, Möller D, Zalpour C. Musculoskeletal health complaints and corresponding risk factors among music students. Med Probl Perform Art. 2018;33:166-174.

17. Thabane L, Ma J, Chu R., Cheng J, Ismaila A, Rios LP, ... \& Goldsmith CH. A tutorial on pilot studies: The what, why and how. BMC Medical Research Methodology. 2010;10:1-10. 
18. Johnson JA, Maddigan SL. Performance of the RAND-12 and SF-12 summary scores in type 2 diabetes. Quality of Life Research. 2004;13: 449-456.

19. Shirley Ryan - Ability Lab. Short Form 12 Item (Version 2) Health Survey March 2013. 2013. https://www.sralab.org/rehabilitation-measures/short-form-12-item-version-2-health-survey. Accessed 20 Jun 2019.

20. Osman A, Wong JL, Bagge CL, Freedenthal S, Gutierrez PM, Lozano G. The depression anxiety stress scales-21 (DASS-21): Further examination of dimensions, scale reliability, and correlates. Journal of Clinical Psychology. 2012; doi:10.1002/jclp.21908.

21. Henry JD, Crawford JR. The short-form version of the Depression Anxiety Stress Scales (DASS-21): Construct validity and normative data in a large non-clinical sample. British Journal of Clinical Psychology 2005; doi:10.1348/014466505X29657.

22. Lovibond PF, Lovibond SH. The structure of negative emotional states: Comparison of the Depression Anxiety Stress Scales (DASS) with the Beck Depression and Anxiety Inventories. Behaviour Research and Therapy. 1995;33:335-343.

23. Latimer J, Maher CG, Refshauge K, Colaco I. The reliability and validity of the Biering-Sorensen test in asymptomatic subjects and subjects reporting current or previous nonspecific low back pain. Spine. 1999; doi:10.1097/00007632-199910150-00004.

24. Durall CJ, Greene PF, Kernozek TW. A comparison of two isometric tests of trunk flexor endurance. The Journal of Strength and Conditioning Research. 2012; doi:10.1519/JSC.0b013e318237ea1c.

25. Beighton PH, Grahame R, Bird H. Hypermobility of joints. 4th ed. London: Springer; 2012.

26. Brito LBB, de Araújo DSMS, de Araújo CGS. Does flexibility influence the ability to sit and rise from the floor? Am J Phys Med and Rehabil. 2013; doi:10.1097/PHM.0b013e3182744203.

27. Ng, S. S., Fong, S. S., Chan, W. L., Hung, B. K., Chung, R. K., Chim, T. H., ... \& Chung, R. C. The sitting and rising test for assessing people with chronic stroke. Journal of physical therapy science. 2016; doi:1589/jpts.28.1701.

28. Hicks GE, Fritz JM, Delitto A, Mishock J. Interrater reliability of clinical examination measures for identification of lumbar segmental instability. Archives of Phys Med and Rehabil. 2003; doi:10.1016/S0003-9993(03)00365-42003.

29. Kenny D. Epidemiology of music performance anxiety. In: The psychology of music performance anxiety, 1st ed. Oxford: Oxford University Press; 2011 p. 83-107.

30. Kenny D. Music performance anxiety: Theory, assessment and treatment. Saarbrücken: LAP Lambert Academic Publishing; 2016. p.43-59.

31. Berque P, Gray H, McFadyen A. Development and psychometric evaluation of the Musculoskeletal Pain Intensity and Interference Questionnaire for professional orchestra musicians. Manual Therapy. 2014; doi:10.1016/j.math.2014.05.015.

32. The Musculoskeletal Pain Intensity and Interference Questionnaire for Musicians (MPIIQM) User Guide. Musicians' Health Scotland. 2014. 
https://pdfs.semanticscholar.org/39e4/5e482cf98a1e50cd66fb26594d9641841096.pdf, Accessed 3 Mar 2020.

33. Pallant J. SPSS Survival Manual. $4^{\text {th }}$ Berkshire, Maidenhead: McGraw Hill; 2011.

34. Canadian Society for Exercise Physiology. Canadian Physical Activity Guidelines (n.d.). https://csepguidelines.ca/adults-18-64/. Accessed 22 Aug 2019.

35. McGill SM. Low back disorders: Evidence-based prevention and rehabilitation. Champaign: Human Kinetics; 2015.

36. Hopman WM, Towheed T, Anastassiades T, Tenenhouse A, Poliquin S, Berger C, ... \& Hanley DA, Canadian normative data for the SF-36 health survey. CMAJ. 2000;163:265-271.

37. Ballenberger N, Möller D, Guptill C, Bruder J, Zalpour C. Occurrence of musculoskeletal health complaints and associated risk factors in music students and non-music students - Interim results from an ongoing cohort study. Presentation at: 2nd World congress on Musician's Physiotherapy, University of Applied Sciences Osnabrueck; 2018 Sept 6-8;Osnabrück.

38. Berque P, Gray H, McFadyen A. Playing-related musculoskeletal problems among professional orchestra musicians in Scotland. Med Probl Perform Art. 2016; doi:10.21091/mppa.2016.2015.

39. Hven L, Frost P, Bonde JPE. Evaluation of pressure pain threshold as a measure of perceived stress and high job strain. PLOS ONE. 2017; doi:10.1371/journal.pone.0167257.

40. Overton M, Du Plessis H, Sole G. Electromyography of neck and shoulder muscles in instrumental musicians with musculoskeletal pain compared to asymptomatic controls: A systematic review and meta-analysis. Musculoskeletal Science and Practice. 2018; doi:10.1016/j.msksp.2018.04.001.

41. Oikawa N, Tsubota S, Chikenji T, Chin G, Aoki M. Wrist positioning and muscle activities in the wrist extensor and flexor during piano playing. Hong Kong Journal of Occupational Therapy. 2018; doi:10.1016/j.hkjot.2011.06.002.

42. Fujii S, Moritani T. Spike shape analysis of surface electromyographic activity in wrist flexor and extensor muscles of the world's fastest drummer. Neuroscience Letters.2012; doi:10.1016/j.neulet.2012.02.089.

43. Díaz-Arribas MJ, Fernández-Serrano M, Royuela A, Kovacs FM, Gallego-lzquierdo T, Ramos-Sánchez M, ... \& Martín-Pariente OS. Minimal clinically important difference in quality of life for patients with low back pain. 2017; doi:1097/BRS.0000000000002298.

44. Nwachukwu BU, Chang B, Voleti PB, Berkanish P, Cohn MR, Altchek DW, ... \& Williams 3rd, RJ. Preoperative short form health survey score is predictive of return to play and minimal clinically important difference at a minimum 2-year follow-up after anterior cruciate ligament reconstruction. The American Journal of Sports Medicine. 2017; doi:10.1177/0363546517714472.

45. Geneen LJ, Moore RA, Clarke C, Martin D, Colvin LA, Smith BH. Physical activity and exercise for chronic pain in adults: An overview of Cochrane Reviews. Cochrane Database of Systematic Reviews. 2017; doi:10.1002/14651858.CD011279.pub3. 
46. Jakobsen MD, Sundstrup E, Brandt M, Andersen LL. Effect of physical exercise on musculoskeletal pain in multiple body regions among healthcare workers: Secondary analysis of a cluster randomized controlled trial. Musculoskeletal Science and Practice. 2018; doi:1016/j.msksp.2018.01.006.

47. Kreutz G, Ginsborg J, Williamon A. Health-promoting behaviours in conservatoire students. Psychology of Music. 2009; doi: 10.1177/0305735607086047.

48. Baadjou VAE, Roussel NA, Verbunt JAMCF, Smeets RJEM, de Bie RA. Systematic review: Risk factors for musculoskeletal disorders in musicians. Occupational Medicine. 2016; doi:10.1093/occmed/kqw052.

49. Ackermann BJ, Kenny DT, O'Brien I, Driscoll TR. Sound Practice-improving occupational health and safety for professional orchestral musicians in Australia. Frontiers in Psychology2014; doi:10.3389/fpsyg.2014.00973.

50. Kenny DT, Davis P, Oates J. Music performance anxiety and occupational stress amongst opera chorus artists and their relationship with state and trait anxiety and perfectionism. Anxiety Disorders.2004;18:757-777.

51. Chesky K, Dawson WJ, Manchester R. Health promotion in schools of music. Med Probl Perform Art.2006;21,142-144.

52. Canadian University Music Society (MusCan). Standing Committee of Institutional Members: Guidelines (Revised May 2014). 2014. https://muscan.org/en/about-us/standing-committee-ofinstitutional-members\#section1. Accessed 31 Mar 2020.

\section{Abbreviations}

PT

OT

MSc

BSc

PhD

CAOT

CAD

GDP

PRMD

ROM
Physiotherapy

Occupational therapy

Master

Bachelor

Doctor of Philosophy

Canadian Association of Occupational Therapists

Canadian Dollar

Gross Domestic Product

Playing Related Musculoskeletal Diseases

Range of Motion 
RAND-12 The 12-question version of the RAND Corporation quality of life measure

ICC Interclass Correlation Coefficient

$\mathrm{SCl} \quad$ Stress-Coping-Inventory

DASS-21 Depression Anxiety Stress Scales, 21-question version

SRT Sitting-Rising-Test

KMPAI-R Kenny Music Performance Anxiety Inventory - Revised

MPIIQM Musculoskeletal Pain Intensity and Interference Questionnaire for Musicians

PA Physical Activity

OR Odds Ratio

MCS Mental Composite Score (of the RAND-12)

PCS Physical Composite Score (of the RAND-12)

\section{Declaration}

\section{Ethics approval and consent to participate}

This study has been approved by the Human Research Ethics Board at the University of Alberta (name: Music Cohort, ID: Pro000673590). All participants gave written, informed consent before the start of testing and the protocol adhered to ethical standards for human subjects.

\section{Consent for Publication}

Not applicable

\section{Availability of data and materials}

Data for this study contains sensitive health information and is unfortunately not available to share outside of the research team, as we do not have ethics approval to share the data.

\section{Competing interests and funding}

This study did not receive any external funding. No other financial arrangements or organizational affiliations are present and there are no competing interests.

\section{Acknowledgements}


Interim reports of this study were presented at the Canadian Association of Occupational Therapists (CAOT) Annual Conference 2018 in Vancouver and at the CAOT Annual Conference 2019 in Niagara Falls. A poster was presented at the Performing Arts Medicine Association annual symposium in 2018 in Orange, CA. This manuscript has not been published nor is currently submitted for publication elsewhere.

All authors have approved the manuscript for submission.

\section{Figures}

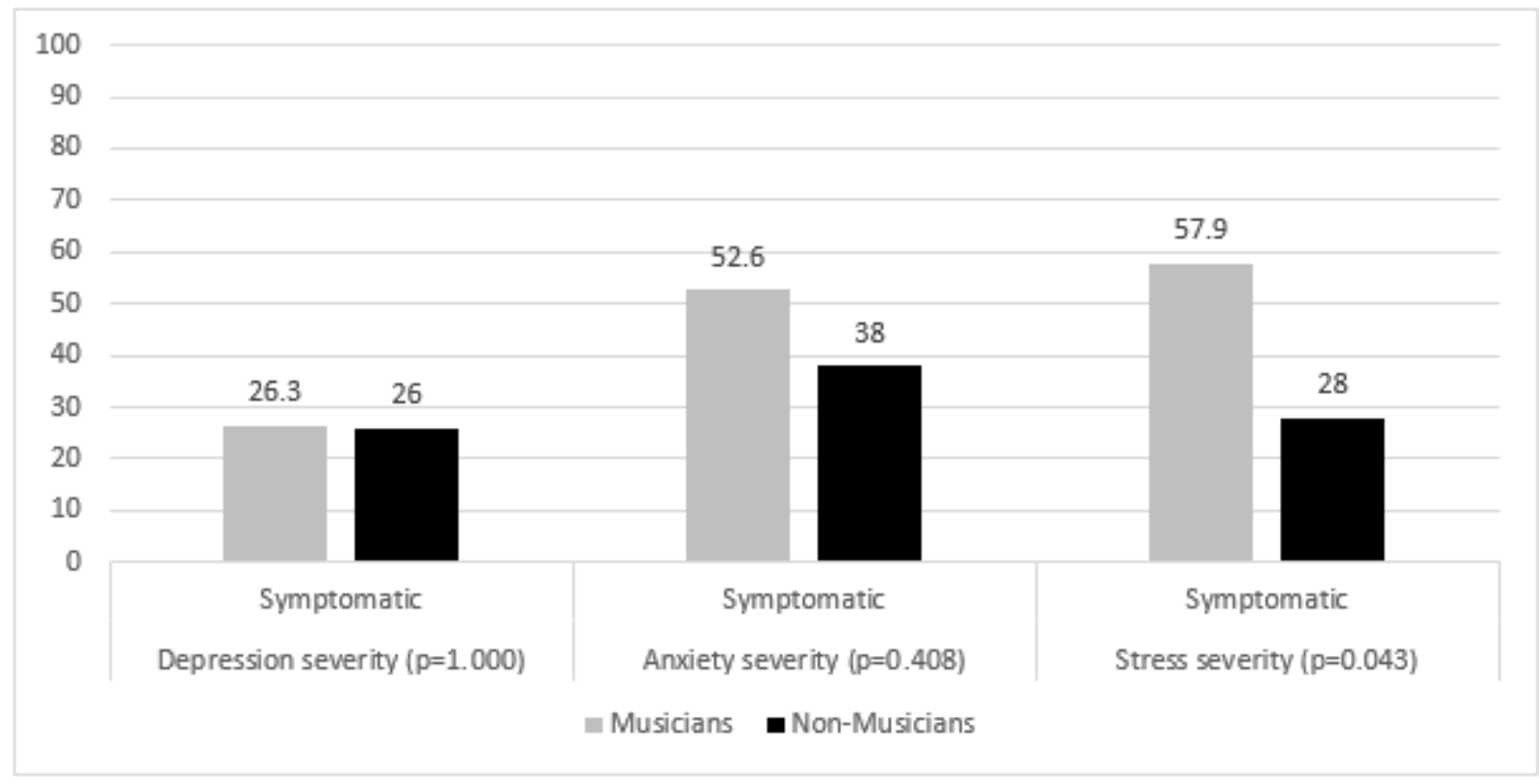

\section{Figure 1}

Frequency (\%) of symptomatic participants by DASS21 domain.

\section{Supplementary Files}

This is a list of supplementary files associated with this preprint. Click to download.

- CONSORTcompleted.docx 